\title{
Preface to the Special Issue of Topics in Catalysis on Beyond Near Ambient Pressure X-Ray Photoelectron Spectroscopy
}

\author{
Axel Knop-Gericke $e^{1,2}$
}

Published online: 22 October 2018

(c) Springer Science+Business Media, LLC, part of Springer Nature 2018

This special Issue of Topics in Catalysis is dedicated to large extend to the application of Near Ambient Pressure X-ray Photoelectron Spectroscopy to the investigation of solid-liquid interphase relevant to electrochemical processes. Two approaches have been developed in order to study electrochemically active solid-liquid interphases. One approach is assigned as "dip and pull". The working electrode is dipped into the electrolyte and pulled out somewhat, so that a meniscus is formed at the interface. The area of the meniscus is illuminated by X-rays in that way, that the released photoelectrons originate from the electrode and the electrolyte can be detected. The second approach bases on the application of graphene, which is used as an electron transparent material. A graphene layer mechanically supported on a holey silicon nitride grid is covered by nanoparticles or a thin layer of the electrode material and exposed to the electrolyte at one side, whereas the second side is exposed to the vacuum of the electron analyzer. The active electrode is illuminated by $\mathrm{X}$-rays through the graphene and the released electrons pass the graphene layer, before they will be detected on the vacuum side of the graphene.

Electrochemical processes like the oxygen evolution reactions (OER) are in the focus of various research groups, since this reaction is important for energy storage. Electrolyzer convert electrical energy which is provided by renewable energy sources like solar cells or wind wheels into chemical energy. This is done by water splitting in which hydrogen will be stored in molecules like methanol or ammonia. In particular the anode at which oxygen is formed suffers from corrosion processes, which oxidize the anode

Axel Knop-Gericke

knop@ fhi-berlin.mpg.de

1 Department of Heterogeneous Reactions, MPI for Chemical Energy Conversion, Stiftstraße 34-36, 45413 Mülheim, Germany

2 Department of Inorganic Chemistry, Fritz-Haber-Institute, Faradayweg 4-6, 14195 Berlin, Germany material and create overvoltages decreasing the efficiency of the energy conversion.

Electrolyzer are operated by noble metals like Ir, since they provide low overvoltage and high stability. On the other hand noble metals are very scarce on earth and therefore other materials should be used as electrodes. In order to develop alternative materials the knowledge of the processes occurring at the interface between the electrode and water on a molecular level is a prerequisite.

Near Ambient Pressure X-ray photoelectron spectroscopy is performed in the mbar pressure range which is still a few orders of magnitude below the pressures at which catalytic reactions are performed in chemical industry. The application of closed reaction cells based on supported graphene membranes offers the possibility to measure XP spectra during catalytic reactions at 1 bar.

In this special issue of Topics in Catalysis a collection of contributions is present which goes beyond near ambient pressure XPS studies performed in the mbar pressure regime, by studying catalytic reactions at ambient pressure or introducing investigations of electrochemical reactions at solid/gas-phase or solid/liquid interfaces. It combines review articles, describing the development of electrochemical cells for XPS studies under reaction conditions and research articles. Among them are studies of $\mathrm{Li}-\mathrm{O}_{2}$ and $\mathrm{Na}-\mathrm{O}_{2}$ batteries and perovskite electrodes used in the oxygen exchange reaction and in the oxygen evolution reaction. $\mathrm{Rh} / \mathrm{YSZ}$ catalysts are investigated during the electrochemically promoted ethylene oxidation. The combination of Density Functional Theory (DFT) with XPS is shown to study complex surface chemistry and catalysis.

Very recent efforts are dedicated to the development of setups which provides spatial resolution under electrochemical relevant conditions in addition to X-ray Photoemission electron microscopy. Two contributions are related to this interesting research field. 